\title{
Identification of Partially Unknown System Matrix of Discrete-time Stochastic Systems via Pseudomeasurement Approach
}

\author{
Akio Tanikawa ${ }^{\dagger}$ and Yuichi Sawada ${ }^{\ddagger}$ \\ $†$ Faculty of Information Science and Technology, \\ Osaka Institute of Technology \\ Kitayama, Hirakata-shi, 573-0196, Japan \\ E-mail: tanikawa@is.oit.ac.jp \\ $\ddagger$ Department of Mechanical and System \\ Engineering, \\ Kyoto Institute of Technology \\ Matsugasaki Sakyo, Kyoto 606-8585, Japan \\ E-mail: sawada@kit.ac.jp
}

\begin{abstract}
In this paper, a new identification method of discretetime linear stochastic systems is proposed. We assume that some entries of the system matrix are unknown and propose a new method which identifies those unknown entries and the state vector of the system simultaneously. The key idea of the proposed method is the use of pseudomeasurement which is a fictitious observation process on the unknown entries and has been introduced by Kameyama and Ohsumi for continuoustime linear stochastic systems. Augmenting the pseudomeasurement with the original observation process, we derive the new identification method by applying the extended Kalman filter.
\end{abstract}

Keywords: system identification, pseudomeasurement, extended Kalman filter.

\section{Introduction}

Identification of stochastic dynamical systems from input and output data has been investigated by many researchers during the past thirty years. One of the most popular methods to identify those systems has been the subspace-based identification method over the last two decades (see [6] and [9]). To recall the idea of the subspace-based method, let us consider the linear system given by

$$
\begin{aligned}
x(t+1) & =A x(t)+B u(t)+w(t), \\
y(t) & =C x(t)+v(t),
\end{aligned}
$$

where $w(t)$ and $v(t)$ are random noise sequences. In the subspace-based method, the identification of the quadruple sytem matrices $(A, B, C)$ is performed from the input and output data $\{u(t), y(t)\}$ within a similarity transformation such as $\left(A_{T}, B_{T}, C_{T}\right)$ where $A_{T}=$ $T^{-1} A T, B_{T}=T^{-1} B, C_{T}=C T$ and $T$ is a transformation matrix. Unfortunately, the matrices $(A, B, C)$

\footnotetext{
${ }^{1}$ Part of this research was supported by the Japan Society for Promotion of Science (JSPS) under Grant-in Aid for Scientific Research (C)-22540158.
}

cannot be identified individually by the subspace-based method because the transformation matrix $T$ cannot be determined.

In this paper, we propose a new identification method of discrete-time linear stochastic systems. Assuming that some entries of the system matrix are unknown, we propose a new method which identifies those unknown entries and the state vector of the system simultaneously. Although it is possible to have an estimate of the matrix $A$ from the estimate of $\left(A_{T}, B_{T}, C_{T}\right)$ by the subspace-based method, we cannot utilize the known part $A_{k}$ of $A$ explicitly. However, in Section 5, we will propose the iterative method which identifies both the states of the system and the unknown part $A_{u}$ of $A$ by making use of $A_{k}$ explicitly. This is one of the advantages of the the proposed method in this paper over the subspace-based method.

The crucial idea of the proposed method is the use of pseudomeasurement which is a fictitious observation process on the unknown entries and has been introduced by Kameyama and Ohsumi in [2] for continuoustime linear stochastic systems for the same purpose as this paper (see [1], [3], [4], [5], [7] and [8] also). Augmenting the pseudomeasurement with the original observation process, we derive the new identification method by applying the extended Kalman filter. Due to the additional observation process data (i.e., pseudomeasurement), we can expect better performance of the new identification method than that of the conventional approach. It can be seen in the simulation results in Section 6.

\section{Problem Statement}

Consider the discrete-time linear stochastic system for $\mathrm{t}=0,1,2, \ldots$ :

$$
\begin{aligned}
x(t+1) & =A x(t)+C u(t)+G w(t), \\
y(t) & =H x(t)+S v(t),
\end{aligned}
$$

where $x(t) \in \mathbb{R}^{n}, y(t) \in \mathbb{R}^{n}$ and $u(t) \in \mathbb{R}^{\ell}$ denote the state vector of the system, the observation vector and the input vector, respectively; $A \in \mathbb{R}^{n \times n}, C \in \mathbb{R}^{n \times \ell}$, $G \in \mathbb{R}^{n \times d_{1}}, H \in \mathbb{R}^{n \times n}, S \in \mathbb{R}^{n \times d_{2}}$. Here, we assume 
that the matrices $C, G, H$ and $S$ are known but the matrix $A$ is partially unknown (see Section 3 for the detail). Moreover, $w(t)\left(\in \mathbb{R}^{d_{1}}\right)$ and $v(t)\left(\in \mathbb{R}^{d_{2}}\right)$ are independent zero mean white Gussian noise sequences with covariance matrices $Q$ and $R$, respectively, i.e., $\mathcal{E}\left\{w(t) w(t)^{T}\right\}=Q$ and $\mathcal{E}\left\{v(t) v(t)^{T}\right\}=R$, where $\mathcal{E}\{\cdot\}$ denotes the mathematical expectation operator and $T$ denotes transposition of a matrix. We assume that the matrix $H$ is nonsingular. Let $Y_{t}$ be the $\sigma$-field $\sigma\{y(s), 0 \leq s \leq t\}$ generated by the observation data $y(s)$ up to the time $t$.

\section{Preliminaries}

Let $A=\left(a_{i j}\right)$ an $n \times n$ matrix in which some of the elements are assumed to be unknown. We separate the unknown entries from $A$ so that

$$
A=A_{k}+A_{u}
$$

holds where the $n \times n$ matrix $A_{k}$ is the known part of $A$ and the $n \times n$ matrix $A_{u}$ is the unknown part of $A$ :

$$
\begin{array}{lll}
\left(A_{k}\right)_{i j}=a_{i j}, & \left(A_{u}\right)_{i j}=0 & \text { if } a_{i j} \text { is known, } \\
\left(A_{k}\right)_{i j}=0, & \left(A_{u}\right)_{i j}=a_{i j} & \text { if } a_{i j} \text { is unknown. }
\end{array}
$$

Here, $(B)_{i j}$ denotes the $(i, j)$-element of the matrix $B$. Assuming that the number of unknown entries is $p$, we define the $p$-dimensional vector $a$ by rearranging $p$ unknown entries $\left\{a_{i j}\right\}$ in the order from upper to lower in the first column and so on. We might write the matrix $A_{u}$ as $A_{u}(a)$ to indicate the unknown parameter vector $a$ explicitly in the following.

Find the $n \times p$ matrix $X(t)$ such that

$$
A_{u}(a) x(t)=X(t) a
$$

holds. Actually, $X(t)$ has the form

$$
X(t)=\left(x(t)^{T} \otimes I_{n}\right) M_{0},
$$

where the symbol $\otimes$ denotes the Kronecker's product of matrices and $M_{0}$ is an $n^{2} \times p$ matrix whose elements are 1 or 0 which is given explicitly in the paper [2] by Kameyama and Ohsumi. From (6)-(7), we can rewrite (3) as

$$
\begin{aligned}
x(t+1)=A_{k} x(t) & +X(t) a+C u(t)+G w(t) \\
=A_{k} x(t) & +\left(x(t)^{T} \otimes I_{n}\right) M_{0} a+C u(t) \\
& +G w(t) .
\end{aligned}
$$

We now try to find a relation between the unknown vector $a$ and the observation process $\{y(t)\}$. First, we have

$$
x(t)=\widetilde{y}(t)-H^{-1} S v(t)
$$

from (4), where we use the notation $\widetilde{y}(t)$ for $H^{-1} y(t)$. It then follows from (8) and (9) that

$$
\widetilde{y}(t+1)=x(t+1)+H^{-1} S v(t+1)
$$

$$
\begin{gathered}
=A_{k} x(t)+\left(x(t)^{T} \otimes I_{n}\right) M_{0} a+C u(t)+G w(t) \\
+H^{-1} S v(t+1) \\
=A_{k} \widetilde{y}(t)+\left(\widetilde{y}(t)^{T} \otimes I_{n}\right) M_{0} a+C u(t)+G w(t) \\
-A_{k} H^{-1} S v(t)-\left\{\left(H^{-1} S v(t)\right)^{T} \otimes I_{n}\right\} M_{0} a \\
+H^{-1} S v(t+1) .
\end{gathered}
$$

We here define the new process

$$
y_{p}(t)=\widetilde{y}(t+1)-A_{k} \widetilde{y}(t)-C u(t)
$$

which is obviously a simple and known function of the input vector $u(t)$ and the observation vectors $y(t)$ and $y(t+1)$. Defining the new matrix

$$
H_{p}(t)=\left(H^{-1} y(t) \otimes I_{n}\right)^{T} M_{0},
$$

we have

$$
\begin{aligned}
y_{p}(t) & =\left(\widetilde{y}(t)^{T} \otimes I_{n}\right)^{T} M_{0} a+(\text { noise terms }) \\
& =H_{p}(t) a+(\text { noise terms }),
\end{aligned}
$$

in view of (10) and (11). We thus have found the relation (13) between $a$ and $y_{p}(t)$. Namely, $\left\{y_{p}(t)\right\}$ can be regarded as the observation process of the unknown vector $a$.

\section{Augmented System}

To identify the unknown vector $a$, we usually consider it as a function $a(t)$ of $t$ and treat it as a random vectorvalued process

$$
a(t+1)=a(t)+G_{a} w_{a}(t),
$$

where $\left\{w_{a}(t)\left(\in \mathbb{R}^{d_{3}}\right)\right\}$ is a newly introduced zero-mean Gaussian white noise sequence with covariance matrix $Q_{a}$ which is independent with the previously given random sequences. Here, the matrices $G_{a}$ and $Q_{a}$ can be preassigned by the users.

Let us introduce a new state vector defined by $z(t):=$ $\left[x(t)^{T}, a(t)^{T}\right]^{T}$. The following augmented system can be obtained from (8) and (14):

$$
z(t+1)=f(z(t))+C_{0} u(t)+G_{0} w_{0}(t)
$$

where

$$
\begin{aligned}
& f(z(t))=\left(f_{1}(z(t)), f_{2}(z(t)), \ldots, f_{n+p}(z(t))\right)^{T} \\
& =\left[\begin{array}{cc}
A_{k} & X(t) \\
O & I
\end{array}\right] z(t) \in \mathbb{R}^{n+p}, \\
& C_{0}=\left[\begin{array}{l}
C \\
O
\end{array}\right] \in \mathbb{R}^{(n+p) \times \ell}, \\
& G_{0}=\left[\begin{array}{cc}
G & O \\
O & G_{a}
\end{array}\right] \in \mathbb{R}^{(n+p) \times\left(d_{1}+d_{3}\right)}, \\
& w_{0}(t)=\left[\begin{array}{c}
w(t) \\
w_{a}(t)
\end{array}\right] \in \mathbb{R}^{d_{1}+d_{3}} .
\end{aligned}
$$


Equality (13) is approximated by the equation

$$
y_{p}(t)=H_{p}(t) a(t)+S_{p} v_{p}(t),
$$

which has the same structure as the observation process (4) and is considered as an additional observation process called pseudomeasurement of the unknown vector $a$. Here, $\left\{v_{p}(t)\left(\in \mathbb{R}^{d_{4}}\right)\right\}$ is a newly introduced zeromean Gaussian white noise sequence with covariance matrix $R_{p}$ which is independent with any previously introduced random sequences. Defining the new vectors $y_{0}(t):=\left[y(t)^{T}, y_{p}(t)^{T}\right]^{T}\left(\in \mathbb{R}^{2 n}\right)$ and $v_{0}(t):=$ $\left[v(t)^{T}, v_{p}(t)^{T}\right]^{T}\left(\in \mathbb{R}^{d_{2}+d_{4}}\right)$, we can obtain the augmented observation process from (4) and (16)

$$
y_{0}(t)=H_{0}(t) z(t)+S_{0} v_{0}(t),
$$

where we used the notations

$$
H_{0}(t)=\left[\begin{array}{cc}
H & O \\
O & H_{p}(t)
\end{array}\right] \in \mathbb{R}^{2 n \times(n+p)}
$$

and

$$
S_{0}=\left[\begin{array}{cc}
S & O \\
O & S_{p}
\end{array}\right] \in \mathbb{R}^{2 n \times\left(d_{2}+d_{4}\right)} .
$$

\section{$5 \quad$ Extended Kalman Filter}

To estimate the state vector $x(t)$ and identify the unknown entries $a$ simultaneously, we can apply the extended Kalman filter to the augmented system (15) and (17) as follows. Denote by $\widehat{z}(t \mid t)$ and $\widehat{z}(t \mid t-1)$, respectively, the least mean square estimates of $z(t)$ with respect to $Y_{t}$ and $Y_{t-1}$. By using the approximation of the nonlinear function $f$ around the estimate $\widehat{z}(t \mid t)$

$$
\begin{aligned}
f(z(t))=f(\widehat{z}(t \mid t)) & +\widehat{F}_{t}(z(t)-\widehat{z}(t \mid t)) \\
& +(\text { higher order terms }),
\end{aligned}
$$

we can obtain the linearized system of (15)

$$
\begin{aligned}
z(t+1)=\widehat{F}_{t} z(t) & +C_{0} u(t)+G_{0} w_{0}(t) \\
& +f(\widehat{z}(t \mid t))-\widehat{F}_{t} \widehat{z}(t \mid t),
\end{aligned}
$$

where

$$
\widehat{F}_{t}=\left(\frac{\partial f(z)}{\partial z^{T}}\right)_{z=\widehat{z}(t \mid t)}=\left[\left(\frac{\partial f^{T}(z)}{\partial z}\right)_{z=\widehat{z}(t \mid t)}\right]^{T}
$$

By applying the Kalman filter to the linear system (17) and (18), we have

$$
\begin{aligned}
& \begin{aligned}
\widehat{z}(t+1 \mid t) & =\widehat{F}_{t} \widehat{z}(t \mid t)+C_{0} u(t)+f(\widehat{z}(t \mid t))-\widehat{F}_{t} \widehat{z}(t \mid t) \\
& =f(\widehat{z}(t \mid t))+C_{0} u(t),
\end{aligned} \\
& \begin{aligned}
\widehat{z}(t+1 \mid t+1) & =\widehat{z}(t+1 \mid t) \\
\quad+K(t+1) & {\left[y_{0}(t+1)-H_{0}(t+1) \widehat{z}(t+1 \mid t)\right], }
\end{aligned}
\end{aligned}
$$

where

$$
\begin{gathered}
K(t+1)=P(t+1 \mid t) H_{0}^{T}(t+1) \\
{\left[H_{0}(t+1) P(t+1 \mid t) H_{0}^{T}(t+1)+S_{0} R_{0} S_{0}^{T}\right]^{-1}} \\
P(t \mid t)=\mathcal{E}\left[\{z(t)-\widehat{z}(t \mid t)\}\{z(t)-\widehat{z}(t \mid t)\}^{T}\right] \\
P(t+1 \mid t)=\mathcal{E}[\{z(t+1)-\widehat{z}(t+1 \mid t)\} \\
\left.\{z(t+1)-\widehat{z}(t+1 \mid t)\}^{T}\right] \\
R_{0}=\left[\begin{array}{cc}
R & O \\
O & R_{p}
\end{array}\right] \in \mathbb{R}^{\left(d_{2}+d_{4}\right) \times\left(d_{2}+d_{4}\right)}
\end{gathered}
$$

Here, $\widehat{z}(0 \mid-1)=\bar{z}_{0}$ is the initial estimate, the matrices $P(t+1 \mid t)$ and $P(t \mid t)$ satisfy the recursive equalities

$$
\begin{aligned}
& P(t+1 \mid t)=\widehat{F}_{t} P(t \mid t) \widehat{F}_{t}^{T}+G_{0} Q_{0} G_{0}^{T}, \\
& P(t \mid t)=P(t \mid t-1)-K(t) H_{0}(t) P(t \mid t-1),
\end{aligned}
$$

respectively, and $P(0 \mid-1)$ is chosen to be $c I$ for some $c(>0)$, where

$$
Q_{0}=\left[\begin{array}{cc}
Q & O \\
O & Q_{p}
\end{array}\right] \in \mathbb{R}^{\left(d_{1}+d_{3}\right) \times\left(d_{1}+d_{3}\right)} .
$$

Let us compute $f(\widehat{z}(t \mid t))$ and $\widehat{F}_{t}$ explicitly. Since

$$
\begin{aligned}
f(z(t)) & =\left[\begin{array}{cc}
A_{k} & X(t) \\
O & I
\end{array}\right]\left[\begin{array}{l}
x(t) \\
a(t)
\end{array}\right] \\
& =\left[\begin{array}{c}
\left\{A_{k}+A_{u}(a(t))\right\} x(t) \\
a(t)
\end{array}\right],
\end{aligned}
$$

we have

$$
\begin{aligned}
f(\widehat{z}(t \mid t)) & =\left[\begin{array}{c}
\left\{A_{k}+A_{u}(\widehat{a}(t \mid t))\right\} \widehat{x}(t \mid t) \\
\widehat{a}(t \mid t)
\end{array}\right] \\
& =\left[\begin{array}{c}
\widehat{A}(t \mid t) \widehat{x}(t \mid t) \\
\widehat{a}(t \mid t)
\end{array}\right],
\end{aligned}
$$

where $\widehat{A}(t \mid t)=A_{k}+A_{u}(\widehat{a}(t \mid t))$.

Notice that

$$
\begin{aligned}
& \frac{\partial f^{T}(z)}{\partial z}=\frac{\partial}{\partial z}\left[x^{T}\left\{A_{k}+A_{u}(a)\right\}^{T} \quad a^{T}\right] \\
& =\left[\begin{array}{cc}
\left\{A_{k}+A_{u}(a)\right\}^{T} & 0 \\
X^{T} & I
\end{array}\right],
\end{aligned}
$$

where we used the equality

$$
\frac{\partial}{\partial a} x^{T} A_{u}^{T}(a)=\frac{\partial}{\partial a} a^{T} X^{T}=X^{T} .
$$

We thus have

$$
\widehat{F}_{t}=\left(\frac{\partial f(z)}{\partial z^{T}}\right)_{z=\widehat{z}(t \mid t)}=\left[\begin{array}{cc}
\widehat{A}(t \mid t) & \widehat{X}(t \mid t) \\
O & I
\end{array}\right],
$$

where

$$
\widehat{X}(t \mid t)=\left.X(t)\right|_{x=\widehat{x}}=\sum_{i=1}^{n} \widehat{x}_{i}(t \mid t) M_{i} .
$$


Hence, we have

$$
\begin{aligned}
& {\left[\begin{array}{l}
\widehat{x}(t+1 \mid t) \\
\widehat{a}(t+1 \mid t)
\end{array}\right]} \\
& =\left[\begin{array}{c}
A_{k} \widehat{x}(t \mid t)+\left(\widehat{x}^{T}(t \mid t) \otimes I_{n}\right) M_{0} \widehat{a}(t \mid t)+C u(t) \\
\widehat{a}(t \mid t)
\end{array}\right]
\end{aligned}
$$

and

$$
\begin{aligned}
& {\left[\begin{array}{l}
\widehat{x}(t+1 \mid t+1) \\
\widehat{a}(t+1 \mid t+1)
\end{array}\right]=\left[\begin{array}{c}
\widehat{x}(t+1 \mid t) \\
\widehat{a}(t+1 \mid t)
\end{array}\right]} \\
& \quad+K(t+1)\left[\begin{array}{c}
y(t+1)-H \widehat{x}(t+1 \mid t) \\
y_{p}(t+1)-H_{p}(t+1) \hat{a}(t+1 \mid t)
\end{array}\right] .
\end{aligned}
$$

\section{Numerical Simulation}

We applied the new identification method via pseudomeasurement approach and the conventional identification method (without pseudomeasurement) to simple examples and compared the results. We consider the single-input two-output two-dimensional linear system with

$$
\begin{aligned}
& A=\left[\begin{array}{cc}
0 & 1 \\
a_{1} & a_{2}
\end{array}\right], \quad C=\left[\begin{array}{l}
0 \\
1
\end{array}\right], \quad G=I_{2}, \\
& H=I_{2}, \quad S=I_{2}, \quad Q=I_{2}, \quad R=0.01 I_{2},
\end{aligned}
$$

where the elements $a_{1}$ and $a_{2}$ of $A$ are unknown. We assign the true parameters $a$ as follows: $a=\left[a_{1}, a_{2}\right]^{T}=$ $[0.8,0.1]^{T}$ and call this example Case 1 . In the simulation, the initial state of system (3) is given by $x(0)=[1,1]^{T}$, and the known input is given by $u(t) \equiv$ 10. We applied the identification method via pseudomeasurement approach starting from the initial estimates as follows: $\widehat{x}(0 \mid-1)=[0,0]^{T}, \widehat{a}(0 \mid-1)=$ $[1,1]^{T}, P(0 \mid-1)=I_{4}$. User-defined parameters are given by $G_{a}=I_{2}, S_{p}=I_{2}, Q_{a}=I_{2}$ and $R_{p}=10 I_{2}$.

The simulation results are shown in Figs. 1-3. While Fig. 1 depicts the trajectory of stochastic system (3), the state estimate $\widehat{x}(t \mid t)$ and the identified parameters $\widehat{a}(t \mid t)$ via pseudomeasurement are shown in Fig. 2 and Fig. 3, respectively. For comparison, we show the simulation results in Figs. 4-6 by the conventional identification method using the extended Kalman filter (without pseudomeasurement), i.e., Fig. 4 for the state trajectory $x(t)$ of $(3)$, Fig. 5 for the state estimate of $x(t)$ and Fig. 6 for the identication of $a$. In view of Fig. 1, Fig. 2, Fig. 4 and Fig. 5, there is no big difference between the two methods on the state estimation. However, we can see the difference between Fig. 3 and Fig. 6. Namely, the identified parameters $\widehat{a}(t \mid t)$ via pseudomeasurement in Fig. 3 converges to the true parameters $a$ faster than those by the conventional method in Fig. 6 .

The second example (called Case 2) is given by the same linear system as Case 1 with the matrix $A$ replaced by

$$
A=\left[\begin{array}{cc}
a_{1} & 1 \\
0 & a_{2}
\end{array}\right]
$$

where the other matrices have the same forms as Case 1. We assign the true parameters $a$ as follows: $a=$ $\left[a_{1}, a_{2}\right]^{T}=[-0.4,0.7]^{T}$. In the simulation, the initial state of system $(3)$ is given by $x(0)=[1,1]^{T}$, and the known input is given by $u(t) \equiv 10$. We applied the identification method via pseudomeasurement approach starting from the initial estimates as follows: $\widehat{x}(0 \mid-1)=[0,0]^{T}, \widehat{a}(0 \mid-1)=[1,-1]^{T}, P(0 \mid-1)=I_{4}$. User-defined parameters are given by the same matrices as Case 1.

The simulation results are shown in Figs. 7-12. While Fig. 7 depicts the trajectory of stochastic system (3), the state estimate $\widehat{x}(t \mid t)$ and the identified parameters $\widehat{a}(t \mid t)$ via pseudomeasurement are shown in Fig. 8 and Fig. 9, respectively. In comparison with the method via pseudomeasurement, the trajectory $x(t)$ of (3), the state estimate of $x(t)$ and the identication of $a$ by the conventional identication method using the extended Kalman filter (without pseudomeasurement) are shown in Fig. 10, Fig.11 and Fig. 12, respectively. In view of Figs. 7-12, we cannot find a big difference between the results of the two methods. But it seems that the behavior of the identication of $a$ by the conventional identication method (without pseudomeasurement) in Fig. 12 is less stable than that of $\widehat{a}(t \mid t)$ via pseudomeasurement in Fig. 9.

\section{Conclusions}

In this paper, we present a new method which identifies unknown entries of the system matrix $A$ and the state vector of the system simultaneously. The key idea of the proposed method is the use of pseudomeasurement which is a fictitious observation process on the unknown entries and has been introduced by Kameyama and Ohsumi for continuous-time linear stochastic systems. The simulation results show the effectiveness of the proposed method.

\section{Acknowledgements}

The authors would like to thank the referees for their helpful comments in the improvement of this paper.

\section{References}

[1] K. Kameyama and A. Ohsumi: Identification of Unknown Parameters of Linear Systems from Noisy Observation Data Using Pseudomeasurement Approach, Proc. of the SICE Annual Conference on Control Systems, Kumamoto, CD-ROM 185-2-3, 2010.

[2] K. Kameyama and A. Ohsumi: Identification of Partially Unknown System Matrix from Noisy Observation Data via Pseudomeasurement Approach, Proc. of the 42nd ISCIE Int. Symp. on Stochastic 
System Theory and Its Applications, Okayama, pp. 27-32, 2010.

[3] T. Kimura, A. Ohsumi and M. Kono: Identification of Unknown Exogenous Input to Stochastic Linear Systems Using Pseudomeasurements, Trans. ISCIE, Vol. 21, No. 12, pp. 390-399, 2008 (in Japanese).

[4] T. Kimura, A. Ohsumi and M. Kono: Observerbased Identification of Unknown Exogenous Input via Pseudomeasurement Approach, SICE J. of Control, Measurement and System Integration, Vol. 2, No. 3, pp. 184-191, 2009.

[5] A. Ohsumi: An Outlook on the Use of Pseudomeasurement in System Identification, Proc. of the 37th SICE Symp. on Control Systems, Kirishima, pp. 91-96, 2008.

[6] A. Ohsumi, K. Kameyama and K.-I Yamaguchi: Subspace Identification for ContinuousTime Stochastic Systems via Distribution-based Approach, Automatica, Vol.38, No. 2, pp.63-79, 2002.

[7] A. Ohsumi, T. Kimura and M. Kono: Kalman Filter-based Identification of Unknown Exogenous Input of Stochastic Linear Systems via Pseudomeasurement Approach, Int. J. of Innovative Computing, Information and Control, Vol. 5, No. 1, pp. 1-17, 2009.

[8] A. Ohsumi, T. Kimura and M. Kono: A Novel Approach to the Identification of Exogenous Input of Stochastic Systems Using Pseudomeasurement, Proc. of IFAC Symp. SYSID 2009, Saint-Malo, France, pp. 296-301, 2009.

[9] P. Van Overschee and B. De Moor: N4SID: Subspace Algorithms for the Identification of Combined Deterministic-Stochastic Systems, Automatica, Vol.30, No. 1, pp.75-93, 1994.

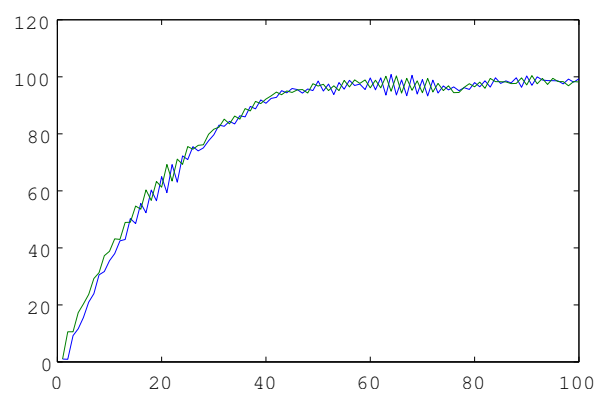

Fig. 1 State trajectory of the linear stochastic system (Case 1)

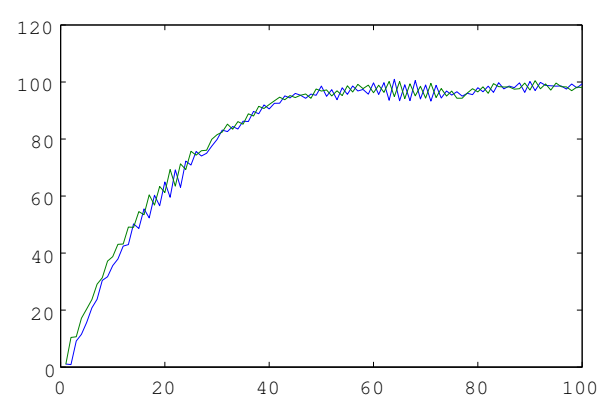

Fig. 2 State estimation for Case 1 via pseudomeasurement

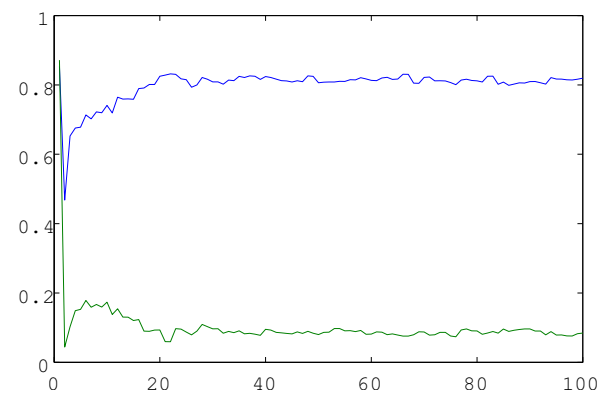

Fig. 3 Identified processes for $a_{1}$ and $a_{2}$ for Case 1 via pseudomeasurement

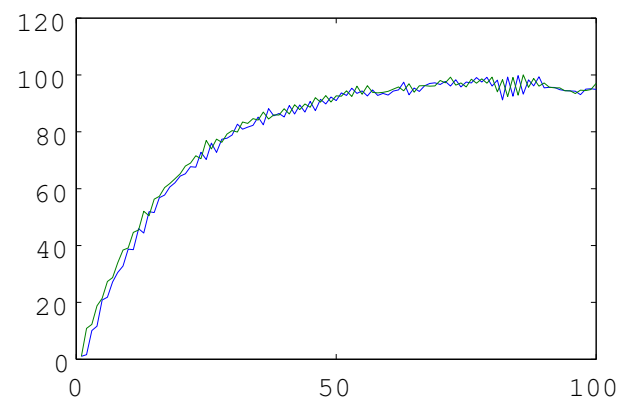

Fig. 4 State trajectory of the linear stochastic system (Case 1)

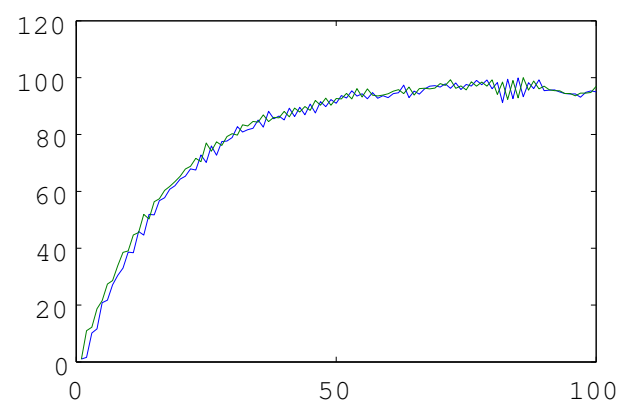

Fig. 5 State estimation for Case 1 without pseudomeasurement 


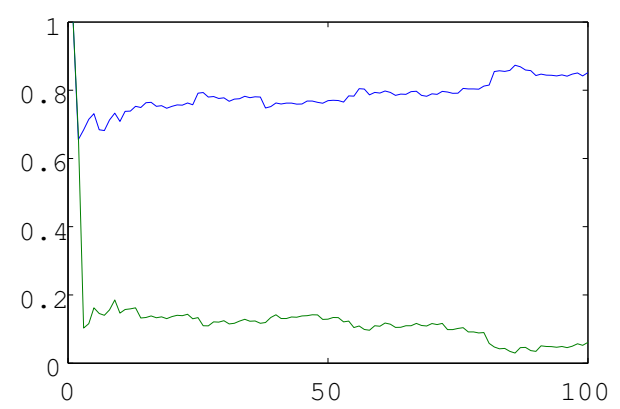

Fig. 6 Identified processes for $a_{1}$ and $a_{2}$ for Case 1 without pseudomeasurement

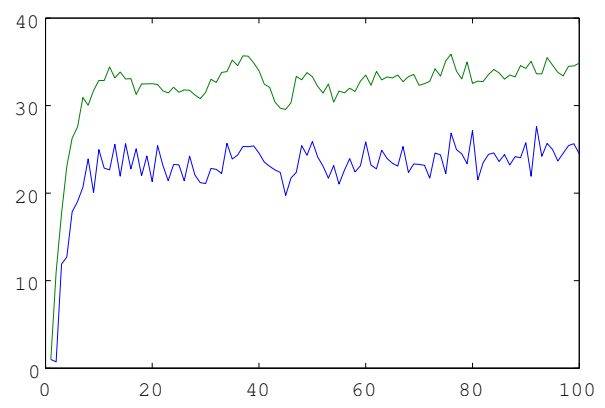

Fig. 7 State trajectory of the linear stochastic process (Case 2)

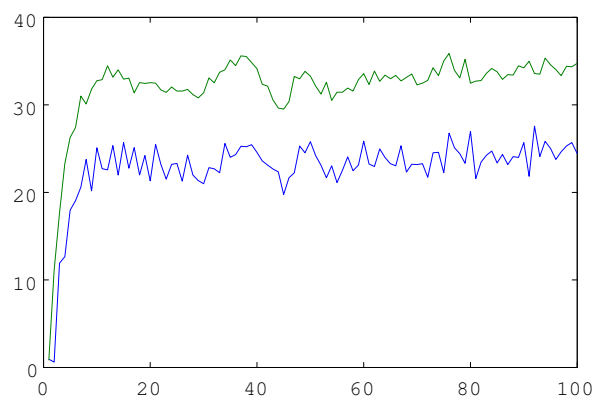

Fig. 8 State estimation for Case 2 via pseudomeasurement

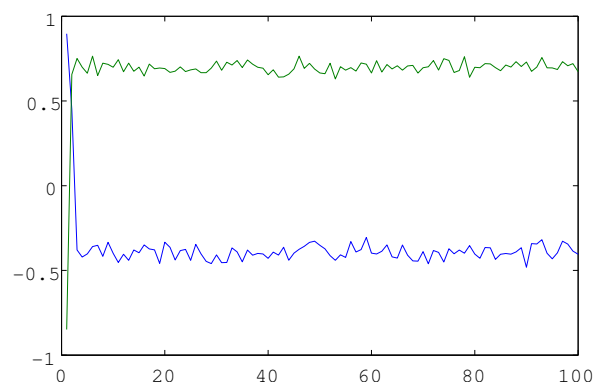

Fig. 9 Identified processes for $a_{1}$ and $a_{2}$ for Case 2 via pseudomeasurement

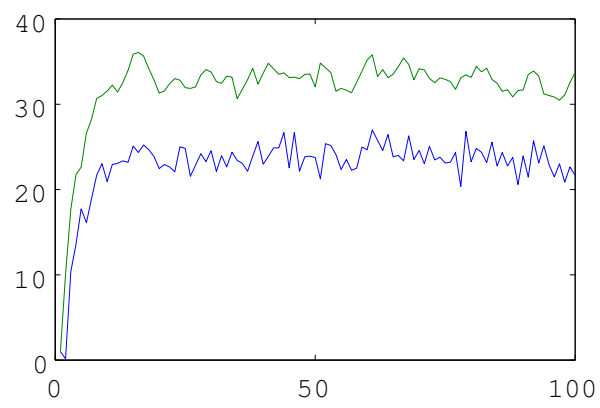

Fig. 10 State trajectory of the linear stochastic process (Case 2)

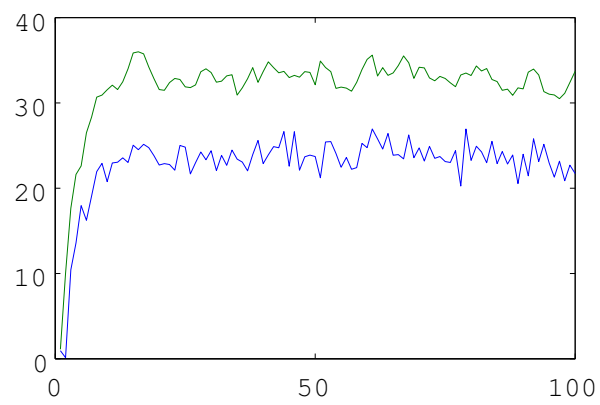

Fig. 11 State estimation for Case 2 without pseudomeasurement

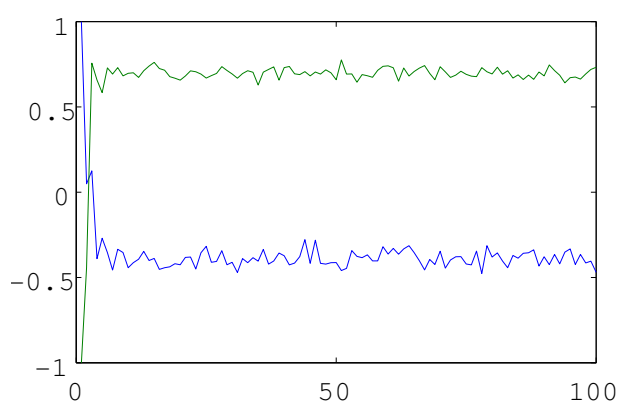

Fig. 12 Identified processes for $a_{1}$ and $a_{2}$ for Case 2 without pseudomeasurement 